Senan Alraho*, Qummar Zaman, and Andreas König

\title{
Wide Programmable Range Fourth-Order, Fully-Differential Sallen-Key MOSFET-C LPF for Impedance Spectroscopy Measurements and Self-X Sensory Electronics in Industry 4.0
}

\author{
Volldifferentieller Sallen-Key-MOSFET-C LPF mit großem programmierbaren Bereich in vierter \\ Ordnung für Impedanzspektroskopie-Messungen und Self-X-Sensorelektronik in Industrie 4.0
}

DOI 10.1515/teme-2021-0064

\begin{abstract}
This paper picks up the need for a wide range programmable corner frequency for anti-aliasing and antiimaging filters in on-chip impedance spectroscopy and sensor signal readout circuitry with self-X properties (selfdiagnosing/healing) for industry 4.0 applications. A fourthorder wide tunable range MOSFET-C low pass filter is designed by using XFAB $0.35 \mu \mathrm{m}$ CMOS technology and Cadence design tools. The proposed circuit is based on fully differential Sallen-Key architecture with Butterworth approximation. It covers a frequency range from $30 \mathrm{~Hz}$ up to $7 \mathrm{MHz}$. Tunability is achieved using a potentially high resistance and linearized configurable MOS resistor to control the filter pole frequency. The configurable elements in the circuit serve as tuning knobs to be controlled by machine learning. The physical design area is $0.39 \mathrm{~mm}^{2}$.
\end{abstract}

Keywords: Self-X properties, impedance spectroscopy, MOSFET-C filter, programmable filter

Zusammenfassung: Dieser Beitrag greift den Bedarf an einer über einen weiten Bereich programmierbaren Eckfrequenz für Anti-Aliasing- und AntiImaging-Filter in On-Chip-Impedanzspektroskopie- und Sensorsignal-Ausleseschaltungen mit Self-X-Eigenschaften (Selbstdiagnose/-heilung) für Industrie 4.0-Anwendungen auf. Ein MOSFET-C-Tiefpassfilter vierter Ordnung mit weitem abstimmbarem Bereich wird unter Verwendung der XFAB 0,35 $\mu \mathrm{m}$ CMOS-Technologie und Cadence DesignTools entworfen. Die vorgeschlagene Schaltung basiert auf einer volldifferenziellen Sallen-Key-Architektur mit

\footnotetext{
*Corresponding author: Senan Alraho, Kognitive Integrierte Sensorsysteme (KISE), TU Kaiserslautern, Deutschland; and College of Electronics Engineering, Ninevah University, Email: aube@eit.uni-kl.de

Qummar Zaman, Andreas König, Kognitive Integrierte Sensorsysteme (KISE), TU Kaiserslautern, Deutschland
}

Butterworth-Approximation. Er deckt einen Frequenzbereich von $30 \mathrm{~Hz}$ bis zu $7 \mathrm{MHz}$ ab. Die Abstimmbarkeit wird durch einen potenziell hochohmigen und linearisierten konfigurierbaren MOS-Widerstand zur Steuerung der Filterpolfrequenz erreicht. Die konfigurierbaren Elemente in der Schaltung dienen als Abstimmknöpfe, die durch maschinelles Lernen gesteuert werden. Die physikalische Designfläche beträgt $0,39 \mathrm{~mm}^{2}$.

Schlüsselwörter: Selbst-X-Eigenschaften, Impedanzspektroskopie, MOSFET-C-Filter, programmierbarer Filter

\section{Introduction}

Impedance spectroscopy (IS) is a versatile and nondestructive characterization technique for inspecting/extracting the electrical parameters of a wide range of materials and compositions with different properties $[6,19]$. IS gained much importance in the measurement and sensors community through its diverse applications, besides its common usage in sensor diagnoses, devices, and circuit characterization $[14,24,26]$. IS approach is based on measuring and analyzing the impedance response of the device under study (DUS) as a function of frequency over a wide span from low sub $\mathrm{Hz}$ up to several $\mathrm{MHz}$, depending on the measurement system capability [21]. Numerous compelling desktop IS equipment (also known as impedance analyzer) are available in the market, e.g., MFIA (Zurich Instruments), E4990A (Keysight Technologies), IM3570 (Hioki), etc. Their main features are the capability in performing the IS measurement under a broad frequency spectrum and covering impedance values from very small to substantial resistance with indubitable measurement accuracy and high speed. However, besides their cost, these equipments are bulky, thus constraining the test in lab condition. Nevertheless, the commercially available chip 
devices like AD5933, AD5940/AD5941, and ADuCM355 from Analog Devices incorporation offer alternative and promising solutions for mobile IS that can be implemented in PCB level with additional circuitries. However, their frequency performance is limited to maximally $200 \mathrm{kHz}$, while numerous applications benefit from a more comprehensive range. Two examples of hand-held on-chip IS from our institute $[25,26]$ extended the IS frequency range from $5 \mathrm{~Hz}$ to $3.5 \mathrm{MHz}$ with an impedance measurement range of about $1 \Omega$ to $8 \mathrm{M} \Omega$. The IS circuitry is further integrated into the implementation of the universal sensor interface with self-X properties (self-calibrating, self-healing) [16] USIX 2.0, functioning as a standalone measurement unit or for the chip diagnoses [13]. One common approach in IS is to apply a small and nondestructive amplitude sinusoidal signal (voltage/current) with a variable frequency of discrete set to the DUS to keep it in the linear or pseudo linear region for the measured voltage-current curve as possible, where the impedance is extracted as the ratio between the AC voltage to the AC current [28]. The low pass filter is an essential part of the IS system. Firstly, it is used as an anti-imaging (reconstruction filter) to smooth the quantized digitally generated sinusoidal stimuli signal $[37,38]$. Secondly, for anti-aliasing the signals in the readout path [26]. Our goal in this project is to improve the on-chip IS quality toward the next generation of USIX 4.0 for industry 4.0 applications. This paperwork focuses on designing the filter unit from the IS system aiming to extend the IS spectrum.

\section{Filter Type Selection}

Four aspects are considered for selecting the appropriate active filter type for the on-chip IS: 1) Continuous-time (CT) or switched capacitor (SC) filter; 2) Bandwidth tunability with accurate and stable quality factor setting over an extensive range of several orders of magnitudes; 3 ) Linearity to reconstruct the sinusoidal stimuli signal in the range of $500 m V_{p-p}$ or more; 4) Area and power consumption. The used instrumentation amplifier (In-Amp) in the planned IS is a CT amplifier; hence it is more compatible with CT filters to avert the clock interference of the SC filters [35]. Two prevalent techniques for realizing active $\mathrm{CT}$ monolithic programmable filters are the active $\mathrm{R} / \mathrm{MOSFET}-\mathrm{C}$ and the transconductance- $\mathrm{C}\left(\mathrm{G}_{\mathrm{m}} \mathrm{C}\right)$ filters. Active $\mathrm{RC}$ filters are the most linear choice for processing large swing signals, gaining their linearity by the use of the closed-loop negative feedback operational amplifier (op-amp) circuits with linear passive elements (Rs and
Cs), which define the filter transfer function [20, 29]. Basically, the magnitude of the loop gain which represents the difference between the open-loop gain $\left(\mathrm{A}_{\mathrm{OL}}\right)$ of the op-amp and the desired closed-loop gain $\left(\mathrm{A}_{\mathrm{CL}}\right)$ of the amplifier circuit must be high enough at the frequency of operation to have a linear transfer function defined by the feedback elements [12]. Furthermore, by considering the peaking in the gain frequency response due to the filter quality factor $(\mathrm{Q})$, a minimum requirement of the op-amp gain-bandwidth product (GBW) is given as [15, 39]:

$$
G B W \geq \kappa \cdot f_{c} \cdot A_{C L} \cdot Q, \quad \kappa: 8 \text { to } 100
$$

where $\mathrm{f}_{\mathrm{C}}$ is the filter cutoff frequency. A higher GBW is a design challenge in CMOS op-amps and requires higher power consumption. Therefore it sets the upper design constraint. Since $\mathrm{f}_{\mathrm{C}}$ is defined by the $\mathrm{RC}$ time constant, the bandwidth tunability can be achieved by using banks of scalable resistors and or capacitors, providing discrete tunability with finite resolution. Fine-tuning implies the use of large banks of small unit sizes [5]. Besides the chip area, a problem arises for the small Rs and Cs values when the on-resistance of the MOSFET selector switch and the parasitic capacitors becomes dominant over the feedback elements, hence degrading the filter linearity and shifting the filter transfer function [23,34]. Nevertheless, for the IS measurement at low $\mathrm{f}_{\mathrm{C}}$ of few $\mathrm{Hz}$, this solution becomes not feasible because it requires vast and impractical passive components size, which cannot be integrated even if the technology supports poly-silicon resistors of extremely high sheet resistance like the XFAB technology used in this paper. $\left(\mathrm{G}_{\mathrm{m}} \mathrm{C}\right)$ filters are open-loop integrators based on un-buffered operational transconductance amplifier (OTA); hence it allows operation at higher $\mathrm{f}_{\mathrm{C}}$ with less power dissipation and area at the cost of signal linearity $[12,29,35]$. The filter time constant and therefore $f_{C}$ is determined by the open-loop amplifier transconductance $\mathrm{g}_{\mathrm{m}}$ and the feedback capacitors, hence this type of filter is more sensitive to the circuit parasitics in comparison to the active closed-loop filters [36]. Arrays of scalable transconductances and capacitors can be used for discrete frequency tunning. Additionally, it is possible to have continuous tuning by controlling the transconductance biasing current $[22,33]$. Linearization techniques are primarily employed to improve the linearity and extend the dynamic range to process large signals [30, 38]. However, the $\left(\mathrm{G}_{\mathrm{m}} \mathrm{C}\right)$ filters may experience performance degradation over the tuning range [8], which is a severe issue in the wide-tunable frequency range required for high-quality IS. Authors in [25] identified practical limitations due to the use of $\left(\mathrm{G}_{\mathrm{m}} \mathrm{C}\right)$ filter in [26]. They accomplished im- 
proved results with MOSFET-C filter replacement, where a tunable range of $(5 \mathrm{~Hz}-3.5 \mathrm{MHz})$ is achieved by using digitally weighted MOS resistors and by controlling the gate voltage of the transistors [2]. In the time domain, MOSFET-C filters still suffer from non-linearity distortion due to the use of the non-linear MOS resistor resulted by the variation of $\mathrm{v}_{\mathrm{gs}}$ with the input signal amplitude. Therefore, MOSFET-C filter is more suitable to process small signals [34]. This paper emphasizes the work on MOSFET-C to attain a wide tunable range and to improve the linearity issue. The remaining considerations for the filter design are filter circuit structure, transfer function approximation, and order degree are covered in the next section.

\section{Proposed Filter Design}

Multiple feedback (MFB) filter topology is a straightforward structure for implementing active low pass R/MOSFET-C fully differential (FD) filters [18, 26]. This type suits very well a filter with fixed bandwidth. However, with variable $f_{C}$ it is difficult to tune the frequency independently from $\mathrm{Q}$ and amplifier gain because the three components are correlated. Under special setting, Tow Thomas filter topology [40] shows possible $\mathrm{f}_{\mathrm{C}}$ tunability but needs two op-amp per biquad and more feedback elements; hence, it consumes more area and power. SallenKey prototype with equal network resistors [15] offer flexible frequency tunability and uses less feedback elements as compared to both MFB and Tow Thomas topologies. However, fully differential implementation requires two single-ended op-amps per biquad [17] or a single differential amplifier with a modified network [31]. The latter solution uses more feedback elements and has complex tunability.

In this paper, we propose a tunable $4^{\text {th }}$ order fully differential Sallen-Key LPF based on the non-inverting fully-balanced differential-difference amplifier (FB-DDF) $[3,4,7]$ as shown in Figure 1. The circuit consists of two biquads with one unity gain buffer connected amplifier per biquad. The buffer configuration has the following advantages; firstly, it offers the most accurate gain setting. Secondly, it requires less GBW per biquad as given in Eq. 1. Thirdly, the input offset voltage $\left(\mathrm{V}_{\mathrm{OS}}\right)$ has no significant effect on the output. Therefore, it relaxes the design from additional offset calibration circuit. Lastly, it provides a least closed-loop output resistance, which leads to more ideal voltage amplifier; thus improves the filter response in the attenuation band at the high frequency region [27]. However, the amplifier input common mode range (ICMR) has to be carefully considered.

Using the filter design coefficient table from [15], the quality factor of the filter is fixed by the capacitor ratios of the individual biquads to perform Butterworth approximation as given below:

$$
\begin{aligned}
& Q_{1}=\frac{1}{2} \sqrt{\frac{C_{2}}{C_{1}}}=0.5412 \\
& Q_{2}=\frac{1}{2} \sqrt{\frac{C_{4}}{C_{3}}}=1.3065
\end{aligned}
$$

and the cutoff frequency of the individual biquads are given by

$$
\begin{aligned}
& f_{C 1}=F S F \cdot f_{C}=\frac{1}{2 \pi R \sqrt{C_{1} C_{2}}} \\
& f_{C 2}=F S F \cdot f_{C}=\frac{1}{2 \pi R \sqrt{C_{3} C_{4}}}
\end{aligned}
$$

where FSF is the frequency scale factor, for Butterworth approximation $\mathrm{FSF}=1$. Since $\mathrm{Q}$ is defined by capacitor ratios, not by absolute values, it is possible to have a stable value by matching them well in the layout. As it is evident by the equations, the filter $\mathrm{f}_{\mathrm{C}}$ can be programmed by varying $\mathrm{R}$ without disturbing the $\mathrm{Q}$. Buterworth approximation features of good amplitude and impulse response. Therefore, the filter is suitable for IS and sensor readout circuitry for conditioning sensor signals with pulse-type characteristics. Contrary, Chebyshev approximation has a sharper transition region at the cost of ripples in the passband and more ringing in the impulse response. Since IS measurement is performed in a discrete set of singlefrequency sinusoidal signals, the ripples in the passband or the pulse response ringing do not affect [37]. However, the filter coefficients requirements for the Chebyshev approximation unnecessarily complicates the design of the proposed circuit and requires more power dissipation due to the higher Q. For the same reason, a higher-order filter is not suggested in this work.

To improve the linearity performance, the linearized high-value tunable bulk-drain connected MOS floating resistor shown in Figure 2 is adopted and modified from [32]. Scalable MOS arrays are used to adjust configurable spans with less constraint on the tuning current $\left(\mathrm{I}_{\text {tune }}\right)$. It is possible to connect several units in series to increase the resistivity and in the same time improve the linearity by reducing the $\mathrm{v}_{\text {sd }}$ dependency [9]. However, for the proposed configurable resistor, this will increase the parasitics in the filter network and shift the filter response. The initial design of the filter capacitors was set smaller. But, due to the interaction with the amplifier input capacitance and the parasitic capacitors of the MOSFET resistors, 


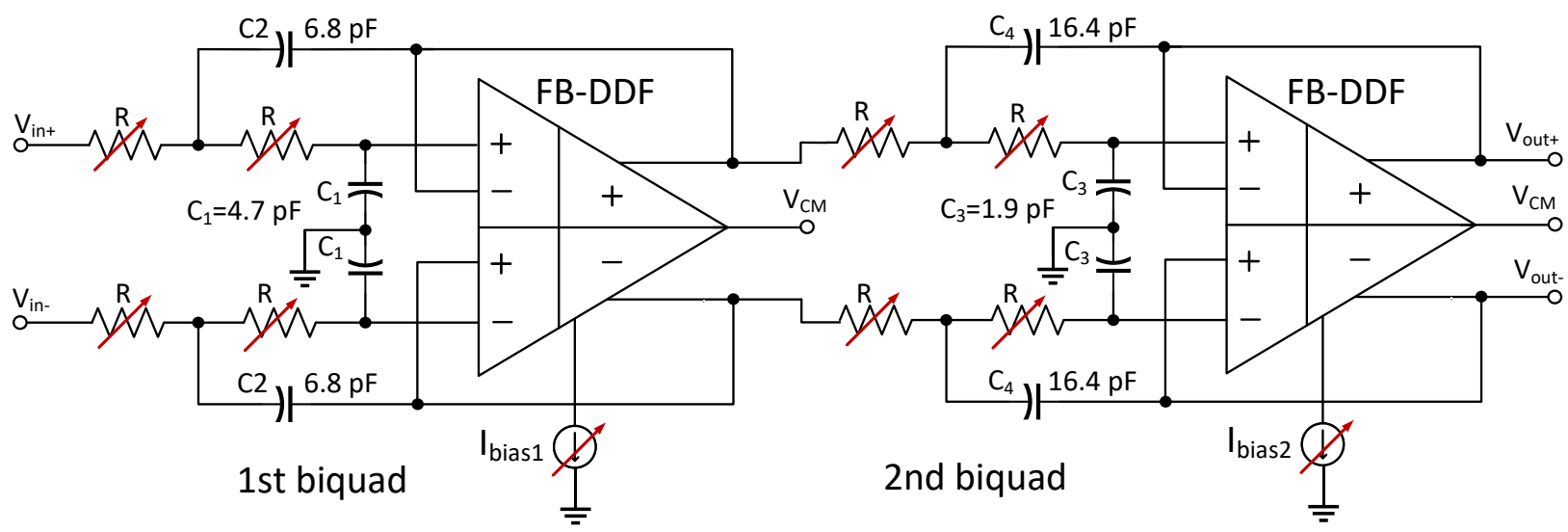

Fig. 1: Proposed programmable fourth order FD Sallen-Key low pass filter.

especially after layout implementation, these values and $\mathrm{Q}$ as well are iterated and increased using the procedure from [34].

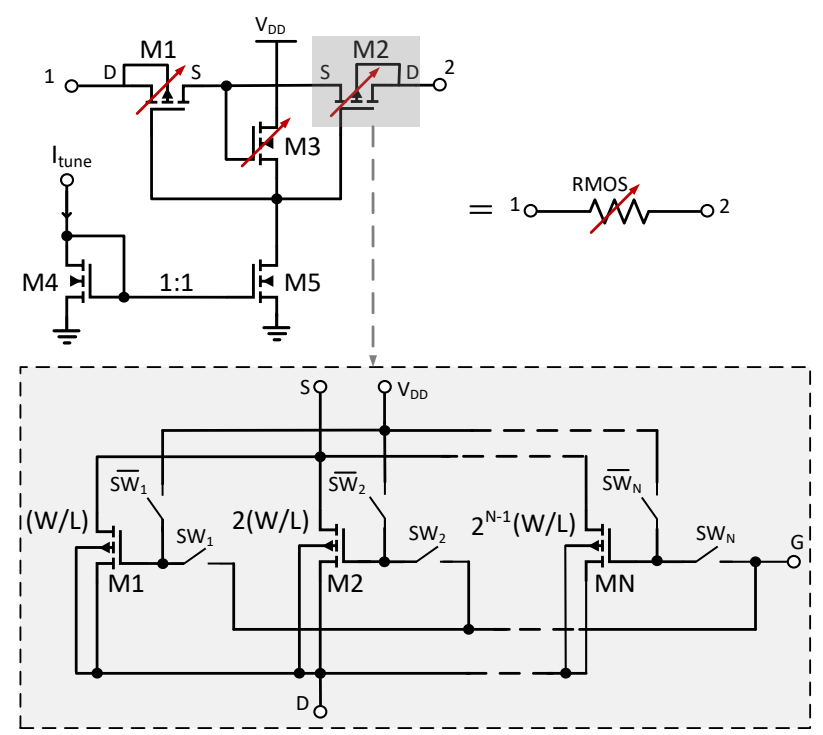

Fig. 2: Proposed configurable floating MOS resistor

Two equal and grounded capacitors $\left(C_{1,2}\right)$ are connected instead of a differentially half-sized connection because the top and bottom plates parasitics are not equal [10]. Consequently, this connection provides better common-mode rejection and also helps to stabilize the common feedback (CMFB) loop of the amplifier [12]. The transistor level design of the FB-DDF is covered in [3]. The core amplifier is realized using the robust class $\mathrm{AB}$ amplifier with rail-to-rail input/output stage [11] to cover the ICMR requirement of the buffered amplifier. The output transistors are designed with minimum channel length and a high biasing current to further decrease the amplifier output resistance. At the same time, this will also decrease the transistor capacitance, hence improving the amplifier stability. The biasing current of the biquads ( $\mathrm{I}_{\mathrm{bias} 1,2}$ ) and hence the GBW can be tuned to the selected $f_{C}$. Therefore, it will save energy at low-frequency operation, while the maximum achieved $G B W$ value is up to $270 \mathrm{MHz}$.

\section{Layout Design and Results}

To take advantages of the fully differential amplifier circuits, care is taken to design a symmetrical layout. Common centroid techniques with the aid of dummy devices are used to improve circuit matching [10]. Design reliability is considered in selecting the width of the wires to avoid material immigration due to the current density limitation of the used metals, which can cause failure after fabrication. The fully custom physical design is presented in Figure 3 with area of $0.39 \mathrm{~mm}^{2}$. Thick metal 3 is used to reduce the supply rails resistivity and provide uniform potential along the rails. Additionally, several decoupling capacitors are used to improve the rail's potential during the AC operation. Figure 4 presents the gain response of the proposed filter for selective values of MOS resistor weights and by varying $\mathrm{I}_{\text {tune }}$ between $100 \mathrm{nA}$ to $30 \mu \mathrm{A}$ with a step size of $100 \mathrm{nA}$. As it can be seen, the $\mathrm{f}_{\mathrm{C}}$ can be adjusted between $30 \mathrm{~Hz}$ up to $7 \mathrm{MHz}$. The achieved total harmonic distortion (THD) is $0.55 \%$ at input signal of $1 \mathrm{v}_{\mathrm{p}-\mathrm{p}}$ and frequency of $1 \mathrm{MHz}$. To examine the stability of the filter, a step signal is applied and the output response is demonstrated in Figure 5. Usually, the Butterworth approximation exhibits a higher overshoot value, however, the proposed filter faces an intermediate drop in the transfer function due to the parasitic effects present at the feedback stage. As a result, the filter roll-off drop 
to $70 \mathrm{~dB} /$ decade. The performance resume of the filter is shown in Table 1 under nominal operating condition. The design is verified and checked against process and voltage variations under industrial temperature range $\left(-40{ }^{\circ} \mathrm{C}\right.$ to $\left.85^{\circ} \mathrm{C}\right)$.

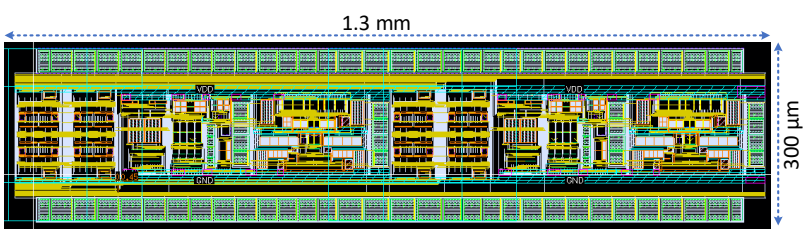

Fig. 3: Layout implementation of the proposed filter.

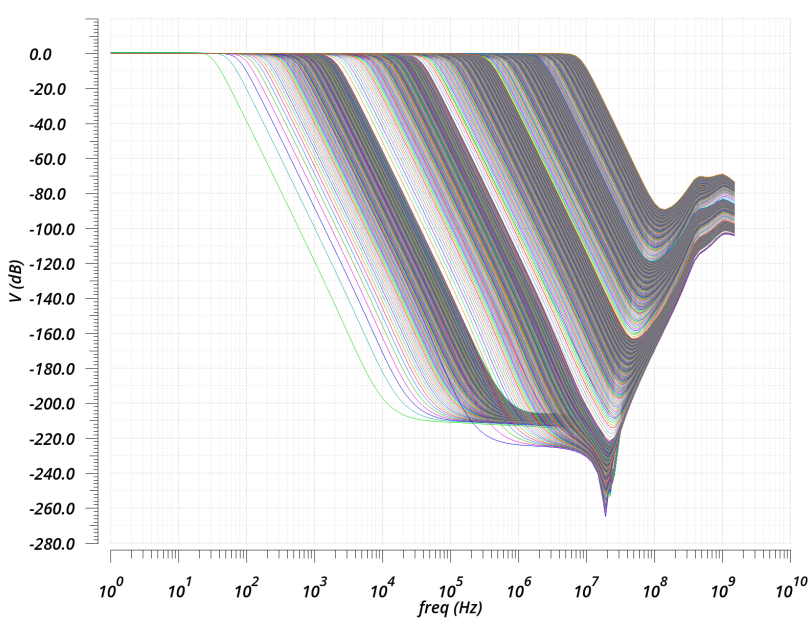

Fig. 4: Gain frequency response of the proposed filter.

Table 1: Post layout performance of the proposed filter, $\mathrm{V}_{\mathrm{DD}}=$ $3.3 \mathrm{~V}, V_{\mathrm{CM}}=1.65 \mathrm{~V}, T=27^{\circ} \mathrm{C}$.

\begin{tabular}{lc}
\hline Parameter & Value \\
\hline DC gain & $0 \mathrm{~dB}$ \\
Power dissipation & $\leq 60 \mathrm{~mW}$ \\
Total harmonic distortion (THD) & $0.55 \%$ \\
@ $V_{I D}=1 V_{P-P}, 1 \mathrm{MHz}$ & \\
Stop band rejection & $-70 \mathrm{~dB}$ \\
Lower $\mathrm{f}_{\mathrm{C}}$ & $30 \mathrm{~Hz}$ \\
Upper $\mathrm{f}_{\mathrm{C}}$ & $7 \mathrm{MHz}$ \\
\hline
\end{tabular}

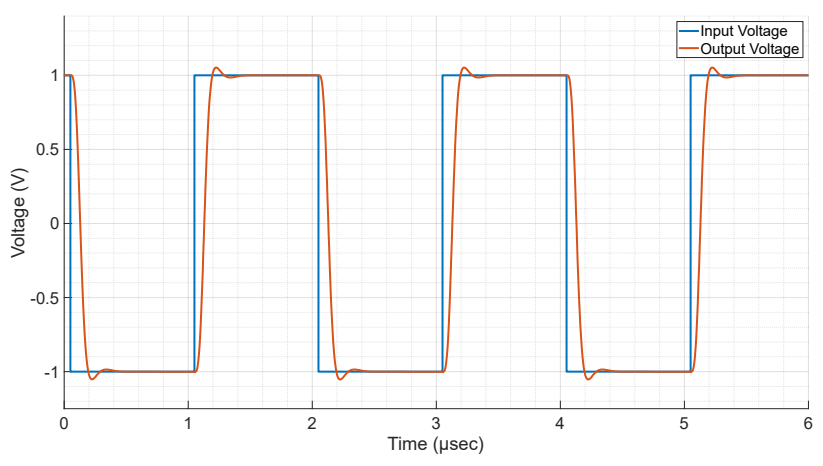

Fig. 5: Impulse response of the proposed filter.

\section{Conclusion and Future Work}

A $4^{\text {th }}$ order fully-differential Sallen-Key low pass filter design is presented in this paper using XFAB $0.35 \mu \mathrm{m}$ CMOS. The wide tunable bandwidth range is achieved using a configurable linearized MOS resistor. With the attained bandwidth, the MOS resistor is equivalent to a variable resistor between $4 \mathrm{k} \Omega$ to $900 \mathrm{M} \Omega$ while the circuit area is only $0.39 \mathrm{~mm}^{2}$. The circuit consumes maximum power of $60 \mathrm{~mW}$, and can be adapted to the selected frequency. The achieved filter specifications satisfies the demand for high-quality IS measurement. For future work, the memristor-based design shall be adopted [1] to replace the current tuning scheme. Low-cost Indirect performance measurement methods will be explored to optimize and tune the filter performance. Furthermore, this filter will be part of the USIX 4.0 for the advanced sensory acquisition platform in industry 4.0 .

\section{References}

[1] H. Abd and A. König. A compact four transistor cmos-design of a floating memristor for adaptive spiking neural networks and corresponding self-x sensor electronics to industry 4.0. tm-Technisches Messen, 87(s1):s91-s96, 2020.

[2] E. Alarcon, H. Martinez, E. Vidal, J. Madrenas, and A. Poveda. D-mrc: Digitally programmable mos resistive circuit. In Proceedings of the 44th IEEE MWSCAS, 2001.

[3] S. Alraho and A. König. Wide input range, fully-differential indirect current feedback instrumentation amplifier for self- $x$ sensory systems. tm-Technisches Messen, 86(s1):62-66, 2019.

[4] H. Alzaher and M. Ismail. A cmos fully balanced differential difference amplifier and its applications. IEEE Transactions on Circuits and Systems II, 2001.

[5] H. Amir-Aslanzadeh, E. J. Pankratz, and E. SanchezSinencio. A 1-v+ $31 \mathrm{dbm}$ iip3, reconfigurable, continuously tunable, power-adjustable active-rc Ipf. IEEE Journal of Solid-State Circuits, 44(2):495-508, 2009. 
[6] E. Barsoukov and J. R. Macdonald. Impedance spectroscopy: theory, experiment, and applications. John Wiley \& Sons, 2018.

[7] H. Elwan, H. Alzaher, and M. Ismail. A new generation of global wireless compatibility. IEEE Circuits and Devices Magazine, 17(1):7-19, 2001

[8] V. Giannini, J. Craninckx, S. D'Amico, and A. Baschirotto. Flexible baseband analog circuits for software-defined radio front-ends. IEEE journal of solid-state circuits, 42(7), 2007.

[9] M. Häberle, D. Djekic, M. Rajabzadeh, G. E. Fantner, K. Lips, M. Ortmanns, and J. Anders. Comparison of different precision pseudo resistor realizations in the dc-feedback of capacitive transimpedance amplifiers. In ICECS 2019. IEEE.

[10] A. Hastings. The art of analog layout. 2006.

[11] R. Hogervorst, J. P. Tero, R. G. Eschauzier, and J. H. Huijsing. A compact power-efficient $3 \vee$ cmos rail-to-rail input/output operational amplifier for vlsi cell libraries. IEEE journal of solid-state circuits, 29(12):1505-1513, 1994.

[12] D. A. Johns and K. Martin. Analog integrated circuit design. John Wiley \& Sons, 2012.

[13] A. C. Kammara, A. Koenig, T. Graef, A. Chinazzo, C. Dobariya, F. Casper, J. Paul, C. Glenske, and J. Traute. Codesign of $\mathrm{mr}$ sensor and sensor electronics for self-x integrated sensory systems. In Sensors and Measuring Systems; 19th ITG/GMA-Symposium, pages 1-4. VDE, 2018.

[14] O. Kanoun. Impedance Spectroscopy: Advanced Applications: Battery Research, Bioimpedance, System Design. De Gruyter, 2019.

[15] J. Karki. Active low-pass filter design. Texas Instruments application report, 2002.

[16] A. Koenig. Integrated sensor electronics with self-x capabilities for advanced sensory systems as a baseline for industry 4.0. In Sensors and Measuring Systems; 19th ITG/GMASymposium, pages 1-4. VDE, 2018.

[17] C. Kolacinski, D. Obrebski, and P. Zagrajek. Integrated, multichannel readout circuit based on chopper amplifier concept for fet-based thz detectors. Circuits, Systems, and Signal Processing, 37(3):984-1006, 2018.

[18] T. Kuehl. Using the infinite-gain, mfb filter topology in fully differential active filters. Texas Instruments application report, 2009.

[19] J. Kusuma and R. G. Balakrishna. A review on electrical characterization techniques performed to study the device performance of quantum dot sensitized solar cells. Solar Energy, 159:682-696, 2018.

[20] F. Lavalle-Aviles and E. Sánchez-Sinencio. A 0.6-v powerefficient active-rc analog low-pass filter with cutoff frequency selection. IEEE Transactions on VLSI Systems, 2020.

[21] J. R. Macdonald. Impedance spectroscopy and its use in analyzing the steady-state ac response of solid and liquid electrolytes. Journal of electroanalytical chemistry and interfacial electrochemistry, 223(1-2):25-50, 1987.

[22] M. Murakawa, S. Yoshizawa, T. Adachi, S. Suzuki, K. Takasuka, M. Iwata, and T. Higuchi. Analogue ehw chip for intermediate frequency filters. In International Conference on Evolvable Systems, pages 134-143. Springer, 1998.

[23] A. T. Phan, R. Farrell, J. Lee, and S.-G. Lee. $380 \mathrm{mhz}$ lowpower sharp-rejection active-rc lpf for ieee 802.15. 4a uwb wpan. In 2009 IEEE International Symposium on Circuits and Systems, pages 1369-1372. IEEE, 2009.

[24] M. Raninec. Overcoming the technical challenges of electrochemical gas sensing. Norwood, MA, USA, Analog Devices, Technical Article, pages 1-5, 2019.

[25] A. Renner, J. Lappas, and A. König. A7. 4-cell optimization for the iisic cmos-chip serving as a front-end for integrated impedance spectroscopy. Proceedings SENSOR 2015.

[26] A. Renner, L. Minghan, and A. Koenig. Architecture and cell design of the iisic cmos-chip serving as a front-end for integrated impedance spectroscopy. In Sensors and Measuring Systems 2014; 17. ITG/GMA Symposium. VDE, 2014.

[27] F. Resta, S. D'Amico, M. De Matteis, and A. Baschirotto. An improved source-follower based sallen-key continuoustime biquadratic cell with auxiliary path. In 2014 NORCHIP, pages 1-4. IEEE, 2014

[28] A. Sacco. Electrochemical impedance spectroscopy: Fundamentals and application in dye-sensitized solar cells. Renewable and Sustainable Energy Reviews, 79:814-829, 2017.

[29] A. C. Sanabria-Borbón and E. Sánchez-Sinencio. Synthesis of high-order continuously tunable low-pass active-r filters. IEEE Transactions on Circuits and Systems I: Regular Papers, 68 (5):1841-1854, 2021.

[30] J. Silva-Martinez, M. S. Steyaert, and W. M. Sansen. A largesignal very low-distortion transconductor for high-frequency continuous-time filters. IEEE Journal of Solid-State Circuits, 26(7):946-955, 1991.

[31] R. P. Singh, K. Layton, and D. J. Comer. A single op amp differential sallen-key biquad. International Journal of Electronics, 100(12):1690-1696, 2013.

[32] A. Tajalli, Y. Leblebici, and E. J. Brauer. Implementing ultra-high-value floating tunable cmos resistors. Electronics letters, 44(5):349-350, 2008.

[33] Y. Tsividis. Continuous-time filters in telecommunications chips. IEEE Communications magazine, 39(4):132-137, 2001.

[34] Y. Tsividis, M. Banu, and J. Khoury. Continuous-time mosfetc filters in vlsi. IEEE Journal of Solid-State Circuits, 21(1): 15-30, 1986.

[35] H. Uhrmann, R. Kolm, and H. Zimmermann. Analog filters in nanometer CMOS, volume 45. Springer Science \& Business Media, 2014.

[36] Y. Wang, L. Ye, H. Liao, R. Huang, and Y. Wang. Highly reconfigurable analog baseband for multistandard wireless receivers in 65-nm cmos. IEEE Transactions on Circuits and Systems II: Express Briefs, 62(3):296-300, 2014.

[37] C.-L. Wei, Y.-W. Wang, and B.-D. Liu. Wide-range filter-based sinusoidal wave synthesizer for electrochemical impedance spectroscopy measurements. IEEE transactions on biomedical circuits and systems, 8(3):442-450, 2013.

[38] C. Yang and A. J. Mason. Fully integrated seven-order frequency-range quadrature sinusoid signal generator. IEEE Transactions on Instrumentation and Measurement, 58(10): 3481-3489, 2009.

[39] L. Ye, C. Shi, H. Liao, R. Huang, and Y. Wang. Highly power-efficient active-rc filters with wide bandwidth-range using low-gain push-pull opamps. IEEE Transactions on Circuits and Systems I: Regular Papers, 60(1):95-107, 2012.

[40] C. Zhang, L. Shang, Y. Wang, and L. Tang. A cmos programmable fourth-order butterworth active-rc low-pass filter. Electronics, 9(2):204, 2020. 